livraisons

d'Histoire

de l'Architecture

\section{Livraisons de l'histoire de l'architecture}

39 | 2020

Maquettes d'architecture

\title{
De la maquette à la modélisation. La recherche architecturale de Frei Otto
}

From model to modeling. Frei Otto's Architectural Research

Vom Modell zum Modellierung. Frei Ottos architektonische Recherche

\section{Georg Vrachliotis}

\section{(2) OpenEdition}

Journals

Édition électronique

URL : http://journals.openedition.org//ha/1575

DOl : $10.4000 /$ /ha. 1575

ISSN : $1960-5994$

Éditeur

Association Livraisons d'histoire de l'architecture - LHA

Édition imprimée

Date de publication : 15 juin 2020

Pagination : 65-75

ISSN : $1627-4970$

Référence électronique

Georg Vrachliotis, « De la maquette à la modélisation. La recherche architecturale de Frei Otto », Livraisons de l'histoire de l'architecture [En ligne], 39 | 2020, mis en ligne le 24 janvier 2021, consulté le 26 janvier 2021. URL : http://journals.openedition.org//ha/1575; DOl : https://doi.org/10.4000/lha. 1575 
Par Georg VRaChLIOTIS ${ }^{1}$

\section{DE LA MAQUETTE À LA MODÉLISATION. LA RECHERCHE ARCHITECTURALE DE FREI OTTO}

"La maquette crée sa propre vérité ", affirme Arthur Drexler, qui fut pendant des années conservateur au département d'architecture du Museum of Modern Art de New York, dans sa contribution "Engineer's Architecture : Truth and Its Consequences ", publiée en 1977 dans le catalogue de l'exposition The Architecture of the École des Beaux-Arts ${ }^{2}$. L'allusion faite ici par Drexler est celle d'une critique nuancée du rôle de la maquette d'architecture qui joue un rôle de plus en plus dominant par rapport à la construction achevée. "Mies van der Rohe, qui autrefois dessinait, a supprimé entre-temps le dessin au profit de maquettes réalistes. Cependant, celles-ci estompent certains détails et présentent une situation parfaite. C'est la raison pour laquelle le vrai bâtiment est en situation d'échec ${ }^{3}$. " Désormais, c'est la maquette plutôt que l'ouvrage construit qui fait irruption sur le devant de la scène. Drexler accusait les architectes modernes d'une perte de réalité causée par la médiatisation excessive de leurs œuvres en maquette. Selon sa thèse, l'architecture d'aujourd'hui se trouve dans une situation comparable à celle jadis de l'École des beaux-arts de Paris. À l'époque, il s'agissait de la prédominance du dessin, aujourd'hui, il s'agit de celle de la maquette.

En matière d'histoire institutionnelle de l'architecture, la critique formulée par Drexler peut sembler tout à fait plausible. On note toutefois sa façon conventionnelle de voir la maquette architecturale. Quelques années avant, il avait non seulement repensé l'interaction historique entre la maquette et le bâtiment, mais aussi remis en question le concept même de maquette. À l'été 1971, soit six ans avant l'exposition sur l'École des beaux-arts, les œuvres expérimentales de Frei Otto furent exposées au Museum of Modern Art, parmi lesquelles une tente blanche flottant dans une cour intérieure. Comme aucun autre architecte du $\mathrm{XX}^{\mathrm{e}}$ siècle ${ }^{4}$, Otto ébranla l'idée traditionnelle de ce que l'on entend par maquette architecturale grâce à la diversité de ses méthodes.

Cela s'exprime dans les différentes destinations des matériaux, outils et dispositifs, ainsi que dans leur couplage opératoire spécifique en tant qu'ensemble de choses,

1. Cet article a été traduit de l'allemand par Hélène Antoni que nous remercions.

2. Arthur Drexler, "Engineer's Architecture: Truth and Its Consequences ", Arthur Drexler (dir.), The Architecture of the École des Beaux-Arts, New York, The Museum of Modern Art, 1977, p. 15.

3. Ibidem.

4. Pour un développement approfondi sur le travail de conception en maquette de Frei Otto, voir Georg Vrachliotis et al. (dir.), Frei Otto. Thinking by Modeling, Spector Books, Leipzig, 2017. 


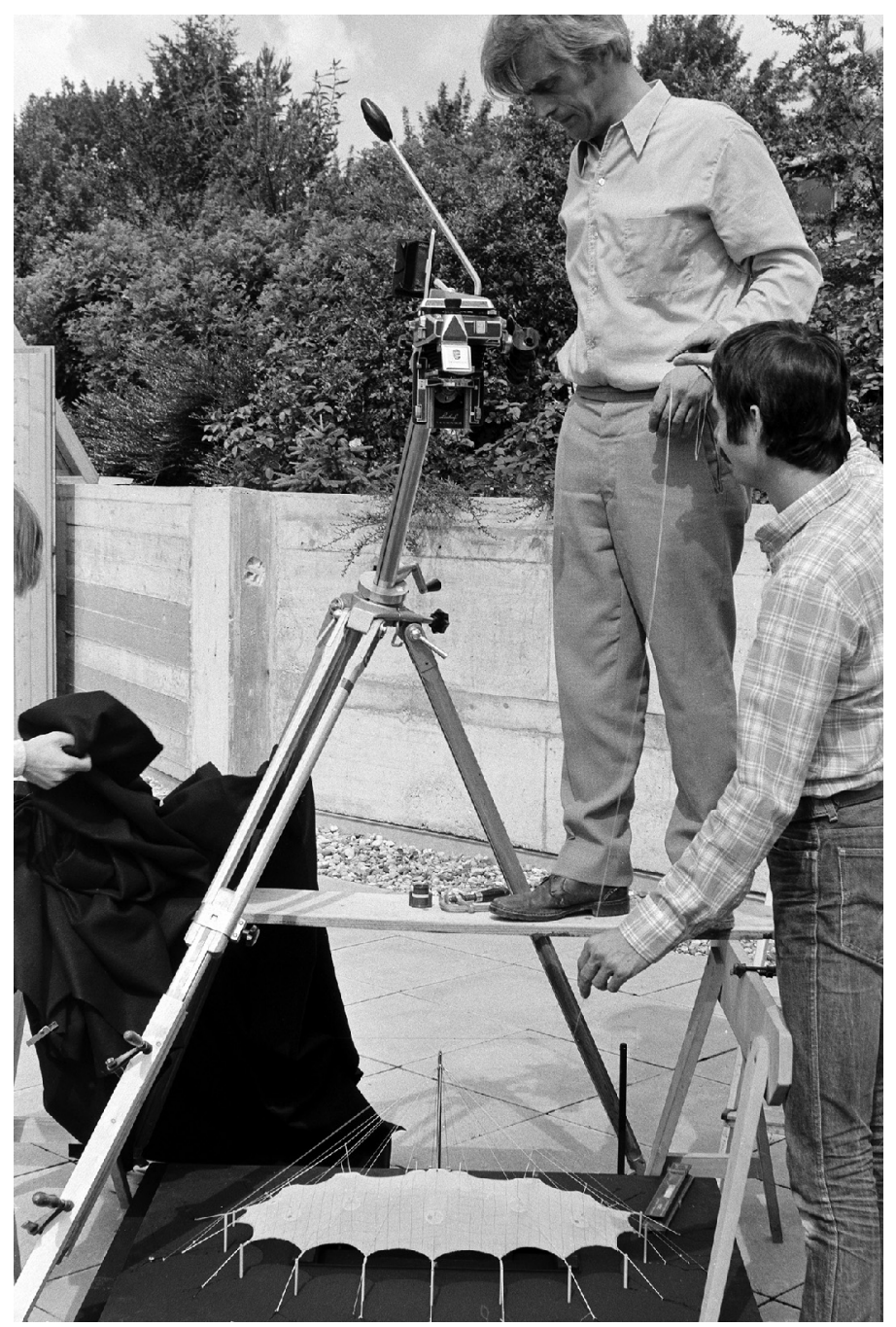

Ill. 1 : Frei Otto photographiant une maquette pour le parc des Jeux Olympiques de Munich, Atelier Warmbronn, vers 1970. (C) saai / Archive for Architecture and Civil Engineering, Karlsruhe Institute for Technology.

c'est-à-dire dans les pratiques techniques, médiatiques et sociales ${ }^{5}$. Une façon de déchiffrer le "code source" de l'architecture expérimentale de Frei Otto est de s'interroger sur la dimension épistémologique de ses maquettes. Relevant aussi bien

5. Voir Gilbert Simondon, Die Existenzweisen technischer Objekte (version originale en français : Du mode d'existence des objets techniques, 1958), Zürich, 2012, en particulier le chapitre "Die technische Erfindung, Grund und Form beim Lebewesen und im erfinderischen Denken », p. 52 et suivantes. 
des champs expérimentaux de l'art et de la science, ses maquettes en filigrane apparaissent souvent comme des agencements d'espace qui essayent de rendre l'invisible mesurable et le visible calculable - de la première petite maquette improvisée en treillis à la maquette éphémère en bulles de savon (ill. 1). Cependant, l'intérêt porté par Otto à l'expérimentation n'est pas basé sur une systématisation technique de l'architecture au sens strict des sciences naturelles. L'accent est plutôt mis sur une interprétation artistique des formes créées expérimentalement à l'aide de paramètres architecturaux. Expérimenter à l'aide de maquettes sert non seulement à étudier les relations de cause à effet, mais aussi, à aller au-delà et à approcher la partie créatrice de formes du processus de conception.

\section{De la construction de maquettes à la construction des nations}

C'est presque au sommet de sa carrière que Frei Otto organisa l'exposition The Work of Frei Otto en 1971 à New York. Après plus de dix ans de recherches personnelles, il est nommé en 1964 à l'Institut des structures légères de l'Université de Stuttgart, créé spécialement pour lui. Avec des architectes, des ingénieurs, des biologistes, des physiciens et des artistes, il en fit un institut de recherche interdisciplinaire à l'échelle mondiale. C'est durant cette période qu'il remporta non seulement le concours pour la conception du pavillon allemand de l'Exposition universelle de Montréal en $1967^{6}$, mais aussi pour l'ensemble des toitures transparentes de l'Olympiapark à Munich en $1972^{7}$. Pour ces deux projets, il s'agissait d'un devoir : celui de développer une architecture nouvelle et innovante pour la jeune République fédérale d'Allemagne. On devait et on voulait opposer le plus grand contraste possible avec l'architecture monumentale du Reich allemand d'Albert Speer. Avec leur ensemble iconique de pavillons transparents pour l'Exposition universelle de Bruxelles en 1958, Egon Eiermann et Sep Ruf avaient déjà montré la voie vers une nouvelle architecture engagée pour l'ouverture au monde (ill. 2).

Pour le pavillon allemand de Montréal, Frei Otto proposa, en collaboration avec l'architecte allemand Rolf Gutbrod, un parcours d'exposition ouvert composé de terrasses spacieuses pour visiteurs, d'une structure en toile de tente flottante, ainsi qu'une petite salle en forme de dôme. La République fédérale se représentait avec une construction expérimentale légère qui, peu de temps après son achèvement, est devenue une favorie des médias internationaux en raison de son symbolisme apparemment heureux. Le terme "Swinging Germany" fit rapidement son chemin ${ }^{8}$.

6. Concernant l'histoire du concours et la réalisation, voir Joachim Kleinmanns, Der deutsche Pavillon der Expo 67 in Montreal. Ein Schlüsselwerk deutscher Nachkriegsarchitektur (Grundlagen, Vol. 89), DOM Publishers, Berlin, 2020.

7. Voir Irene Meissner, «Leichtbau gegen Repräsentation. Von Montreal 67 zu München 72 », Georg Vrachliotis et al. (dir.), Frei Otto. Thinking by Modeling, Spector Books, Leipzig, 2017.

8. Rudolf Leonhardt, "Swinging Germany », Die Zeit, 12 mai 1967. 


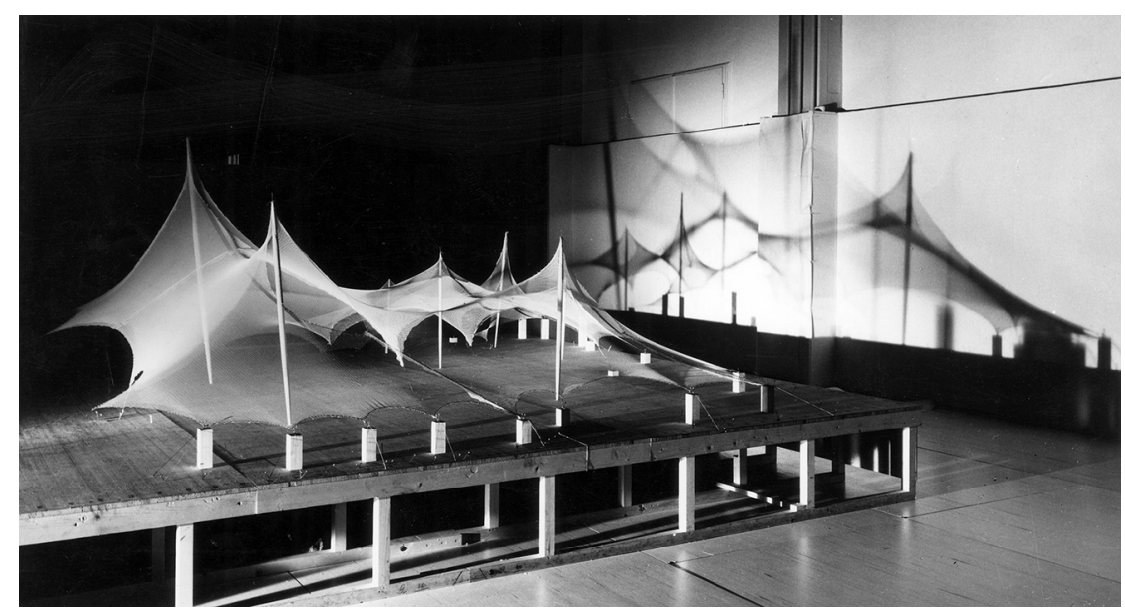

Ill. 2 : Maquette d'étude, pavillon de l'Allemagne de l'Exposition de 1967 à Montréal, Institute for Lightweight Structures (IL) de l'Université de Stuttgart, 1965. (C) saai / Archive for Architecture and Civil Engineering, Karlsruhe Institute for Technology.

La grande tente avait une forme irrégulière et couvrait une superficie d'environ 8000 mètres carrés, soutenue par huit mâts pointant dans des directions différentes. Mais l'histoire fascinante du pavillon commence beaucoup plus tôt. Gutbrod et Otto conçoivent non seulement le pavillon, mais aussi les maquettes, outils, instruments de mesure et méthodes de visualisation nécessaires à la conception et à la planification (ill. 3). En d'autres termes, il s'agit ici d'une reformulation matérielle de la culture technique du projet. La volonté de construire se manifeste sous les traits de l'inventeur. C'est le vrai point fort du projet.

\section{Maquettes éphémères}

Pour la structure du pavillon, Otto utilise la statique de la maquette (Modellstatik) en raison de la grande portée du toit, une méthode de travail empirique issue des sciences de l'ingénieur qui traite de l'interaction des forces entre la structure et la construction directement sur la maquette. En conséquence, la simulation des pressions statiques du vent sur le pavillon est réalisable sur une simple maquette en bois (ill. 4). Afin de tester la stabilité au vent, un modèle de soufflerie en contreplaqué stratifié à l'échelle de 1:150 est construit selon les courbes de niveau de la maquette en toile. 130 fines perforations de la maquette ont permis de mesurer les différences de pression à l'aide de tuyaux en plastique et de tubes en verre partiellement remplis d'alcool. Les variations des niveaux d'alcool dans les tubes en verre pouvaient alors être constatées par la photographie. De cette façon, les conditions d'exposition au vent de la toile complexe de couverture du pavillon pouvaient être 


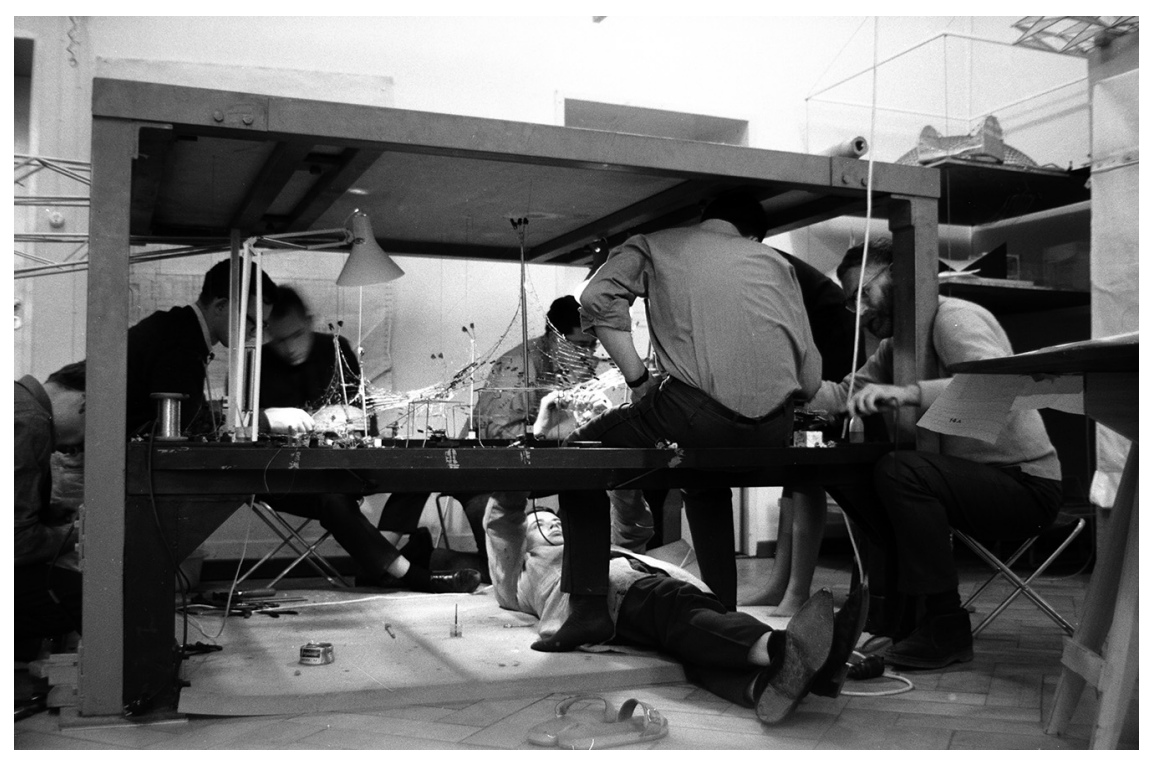

Ill. 3 : Frei Otto et son équipe travaillent sur la grande maquette expérimentale pour le pavillon allemand, exposition de 1967 à Montréal, Institute for Lightweight Structures (IL) de l'Université de Stuttgart, 1965-66. (C) saai / Archive for Architecture and Civil Engineering, Karlsruhe Institute for Technology.

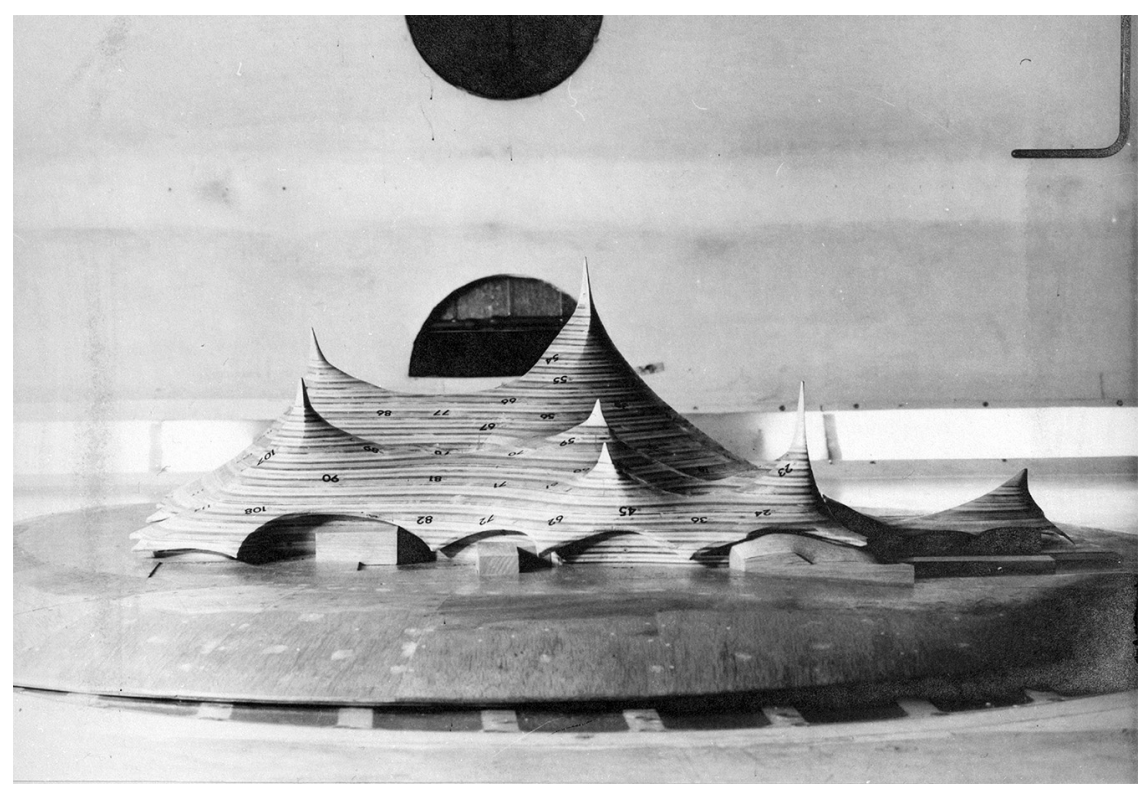

Ill. 4 : Maquette d'une soufflerie en bois pour le pavillon de l'Allemagne, exposition de 1967 à Montréal, Institute for Lightweight Structures (IL) de l'University de Stuttgart, 1965/66. (C) saai / Archive for Architecture and Civil Engineering, Karlsruhe Institute for Technology. 
simulées sur la maquette, et, au final, cartographiées par le dessin. Pour Otto, l'expérimentation sur la maquette vise donc non seulement la forme, mais aussi la mesure graphique et photographique des forces impliquées. Au fil du temps, ce processus de médiatisation technique est devenu si complexe qu'il a ensuite nécessité le développement d'une table de mesure spéciale sur laquelle la maquette pouvait être fixée et l'enregistrement pouvait être entrepris par photogrammétrie à l'aide de caméras spéciales. "La table de mesure est une installation qui permet de déplacer des coordonnées $(\mathrm{x}, \mathrm{y}, \mathrm{z})$ dans un point de mesure. Chaque orientation de coordonnées est représentée par une tige filetée, déplacée chacune par un moteur électrique. Les rotations des tiges sont mesurées. Les valeurs mesurées peuvent être lues sur un afficheur numérique et peuvent être imprimées en clair ou sur bande perforée. Le ruban perforé peut être utilisé pour l'entrée des données dans les systèmes informatiques et les machines à dessiner automatisées. Sur la planche à dessin de la table de mesure, le plan de la maquette peut être reproduit à l'aide d'une aiguille de ponction automatique pendant le mesurage des coordonnées ${ }^{9}$. " De cette façon, il est possible de mesurer précisément l'équilibre des forces de la maquette à l'aide de la photographie et de l'évaluer mathématiquement. Cette démarche s'apparentait à un processus d'élargissement dans lequel l'objet technique était intégré dans un ensemble. En d'autres termes, la maquette et l'appareillage de mesure sont devenus des acteurs du même système d'expérimentation ${ }^{10}$.

\section{"Une bulle de savon est une bulle de savon"}

La façon dont Frei Otto élargit ici radicalement l'interaction de la maquette, de la médiatisation et de la matérialité devient lisible lorsque l'on considère la palette des substances et des matériaux qui ont été utilisés. Dès la fin des années 1950, Otto avait découvert que des membranes très minces et relativement stables peuvent se former à partir d'eau distillée et de quelques gouttes de liquide vaisselle ${ }^{11}$. Aussi inhabituelle que cette méthode puisse paraître, Frei n'était nullement le seul à s'en préoccuper. Dans la petite communauté d'architectes portés sur l'expérimentation technique, bien connectée au niveau international, les travaux sur les propriétés

9. Frei Otto, «Protokoll. Über die Arbeiten des Instituts für Leichte Flächentragwerke an den Modellversuchen und Auswertungen am Projekt Deutscher Pavillon, 1967, Weltausstellung, Montreal ", manuscrit reproduit de l'Institut des structures légères (Instituts für Leichte Flächentragwerke), mars 1967, non paginé.

10. Quelques années plus tard, Frei Otto développera un processus similaire pour l'ensemble des toitures transparentes de l'Olympiapark à Munich (1972) et la voûte en résille de bois de la Multihalle à Mannheim (1975). Voir, Irene Meissner, «Leichtbau gegen Repräsentation. Von Montreal 67 zu München 72 ", Georg Vrachliotis et al. (dir.), Frei Otto, Thinking by Modeling, Spector Books, Leipzig, 2017, p. 41-53; et Georg Vrachliotis, Frei Otto, Carlfried Mutschler. Multihalle, Spector Books, 2017.

11. L'architecte polonais Lech Tomaszewski engagea aussi dans les années 1960 des expérimentations sur les bulles de savon à l'Académie des Beaux-Arts de Varsovie, voir Jola Gola, "Spaces Beyond the Limits of Perception ", Piktogram, 3, no 12, 2008, p. 81. 


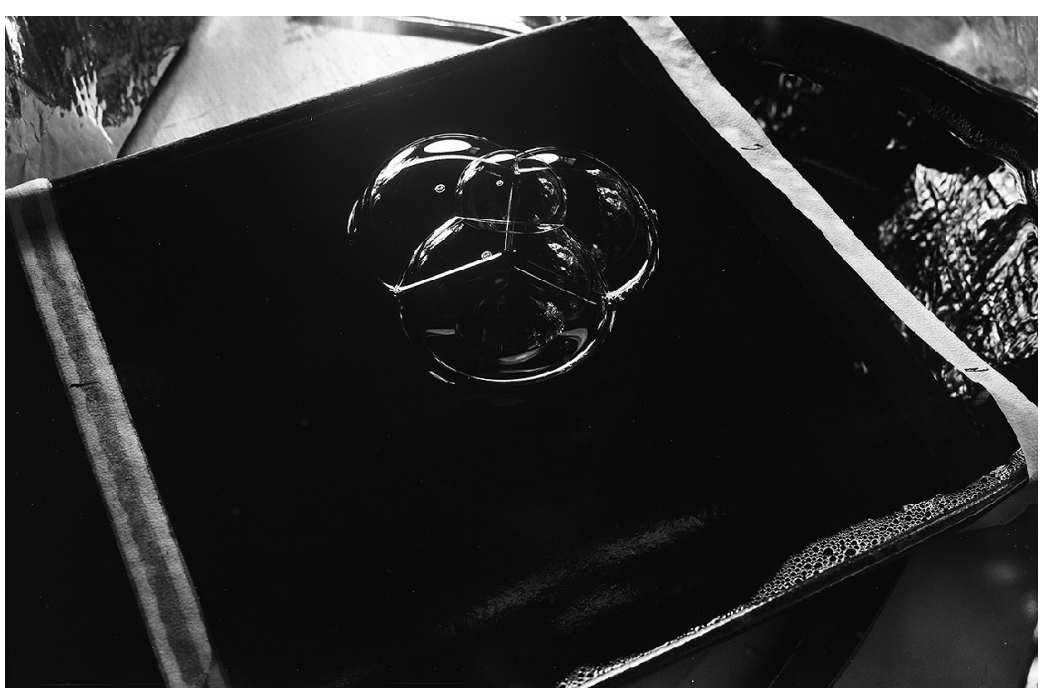

Ill. 5 : Trois bulles de savon de taille égale assemblées pour créer une forme régulière, Atelier Warmbronn. (c) saai / Archive for Architecture and Civil Engineering, Karlsruhe Institute for Technology.

des bulles de savon sont tout à fait d'actualité dans les années 1960 (ill. 5). Par exemple, l'ingénieur d'origine française Robert Le Ricolais utilisait la bulle de savon comme matériau didactique dans ses séminaires à l'Université de Pennsylvanie : au travers de ces expériences sur les films transparents des bulles de savon, ses étudiants devaient mieux appréhender les lois statiques et développer leur imagination ${ }^{12}$. En fait, les maquettes en bulles de savon ne sont pas seulement très parlantes; en tant que modèles fonctionnels, "elles incarnent également la preuve matérielle de leur propre structure porteuse. Une bulle de savon est une bulle de savon et tu sais quand l'expérience fonctionne. " ${ }^{13}$ Lorsque l'on plonge, puis retire un cadre couvert de fil de fer courbé dans une solution savonneuse, une fine pellicule de savon se crée. Si le cadre prend une forme tridimensionnelle, la bulle de savon prend la forme d'une surface incurvée en trois dimensions (ill. 6 et 7). "Avec l'évolution des [...] films tendus, il était fascinant de voir comment, lors de la recherche de formes à construire avec un minimum de matériaux de construction, des formes d'une grande clarté et d'une beauté captivante furent créées. C'étaient des formes qui ne pouvaient pas être conçues par l'homme [... ${ }^{14}$ ». Dans d'innombrables expériences, Otto a pu observer qu'une membrane ainsi construite est principalement déterminée par ses bordures extérieures, c'est-à-dire les points hauts et bas, et possède des propriétés géométriques

12. Voir Robert Le Ricolais, "Structures et formes ", L'Architecture d'aujourd'hui, 30, n 84, 1959, p. 64-68.

13. Citation d'après un échange avec un collaborateur de longue date de Frei Otto, Berthold Burkhardt avec Georg Vrachliotis, Braunschweig, 2019.

14. Frei Otto, Peter Strohmeyer, "Zelte ", Deutsche Bauzeitung, 7, 1960, p. 352. 


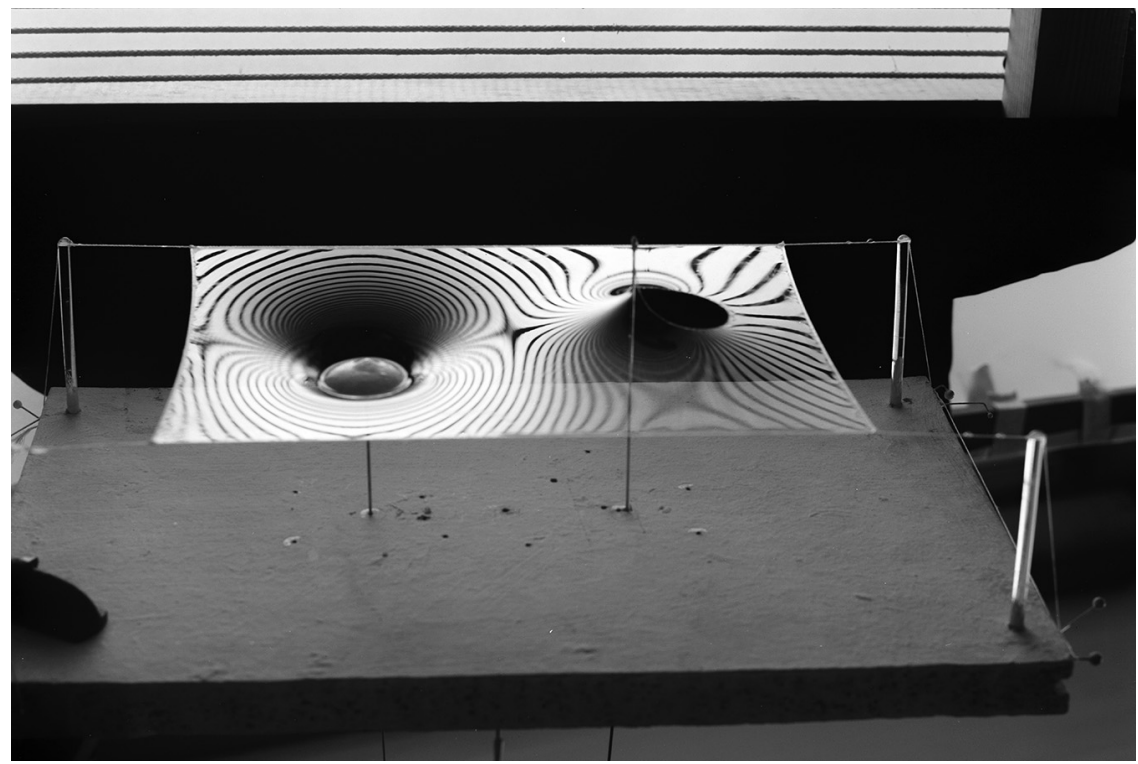

Ill. 6: Maquette en bulles de savon avec des courbes de niveau, Institute for Lightweight Structures (IL) de l'Université de Stuttgart, vers 1965. (C) saai / Archive for Architecture and Civil Engineering, Karlsruhe Institute for Technology.

et physiques spécifiques. La tension superficielle d'une telle membrane est la même en chaque point et dans chaque direction, ce qui crée une stabilité et une efficacité particulières qui conduisent à la formation de surfaces dites minimales ${ }^{15}$.

\section{La maquette comme machine}

Inspiré par l'esthétique et l'efficacité de cette variété de formes, Otto a commencé à développer un certain nombre d'installations de test avec son collaborateur Larry Medlin dès les travaux préparatoires du pavillon allemand de l'Exposition universelle de 1967, en vue de mesurer géométriquement de façon plus précise ces formes de surface minimales. Pour les premières tentatives, il a utilisé un simple cadre ouvert, mais dans les ateliers à l'air sec exposés aux courants d'air, les maquettes très délicates en bulles de savon se volatilisaient toujours. Si on veut enregistrer géométriquement les formes de surface minimales des maquettes en bulles de savon, celles-ci doivent présenter une certaine durabilité. La maquette doit être protégée des mouvements d'air et évoluer dans une atmosphère exempte de poussière, d'un degré d'humidité élevé et en basse température pour empêcher un dessèchement trop rapide.

15. Voir Seifenblasen, Mitteilungen des Instituts für Leichte Flächentragwerke (IL), 18, 1988. 


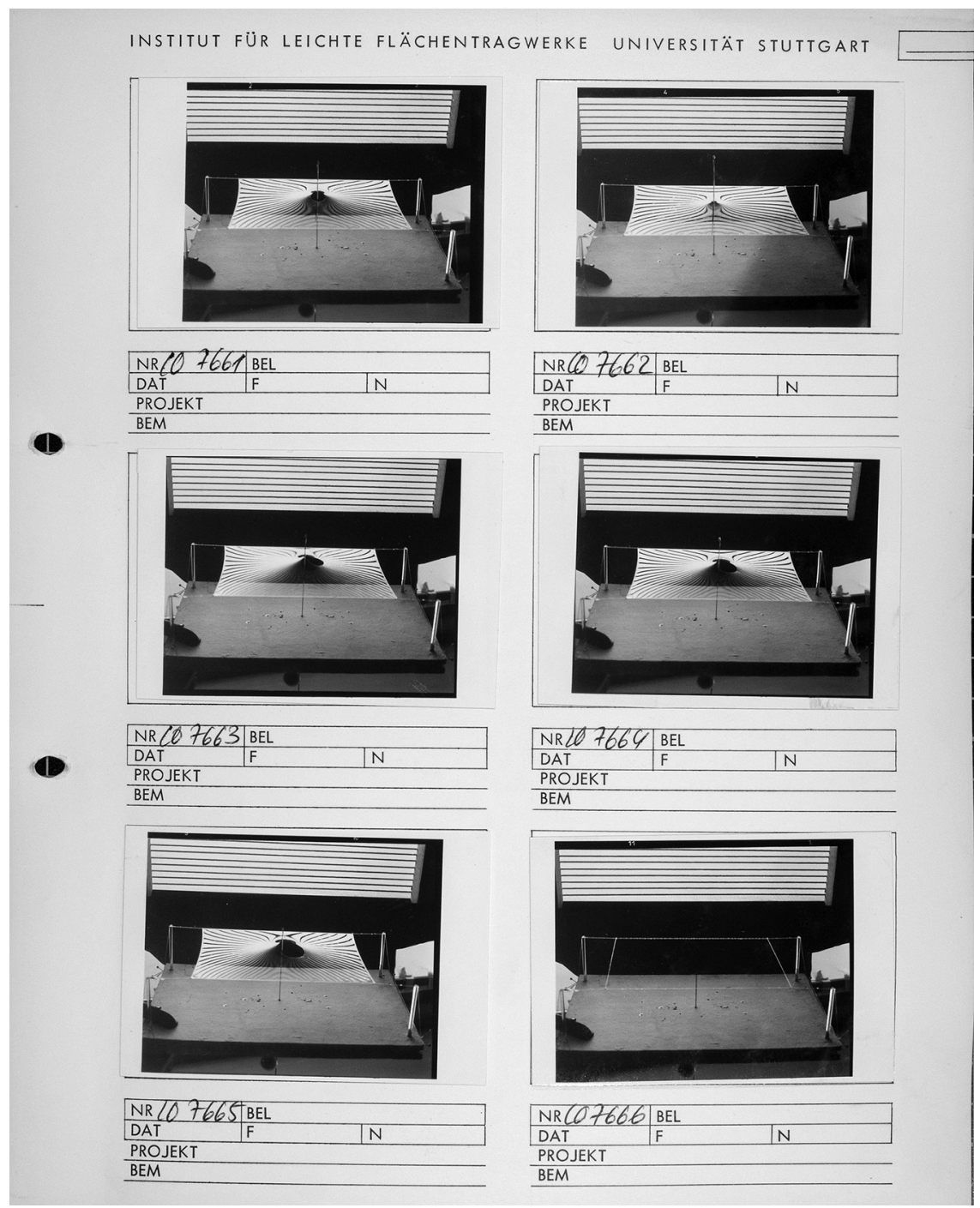

Ill. 7 : Maquettes en bulles de savon, Institute for Lightweight Structures (IL) de l'Université de Stuttgart, planche contact, vers 1965. (C) saai / Archive for Architecture and Civil Engineering, Karlsruhe Institute for Technology.

Au cours des presque quinze années suivantes, ce processus sera continuellement développé et amélioré sur le plan technologique et conceptuel, et l'étendue de ses fonctions élargie, de sorte qu'il était même possible de construire une machine à bulles de savon spéciale, en quelque sorte un dispositif à surfaces minimales. En conséquence, ses composants les plus importants sont une chambre, un dispositif de refroidissement et d'humidification de l'air, une lumière parallèle, une grille de 


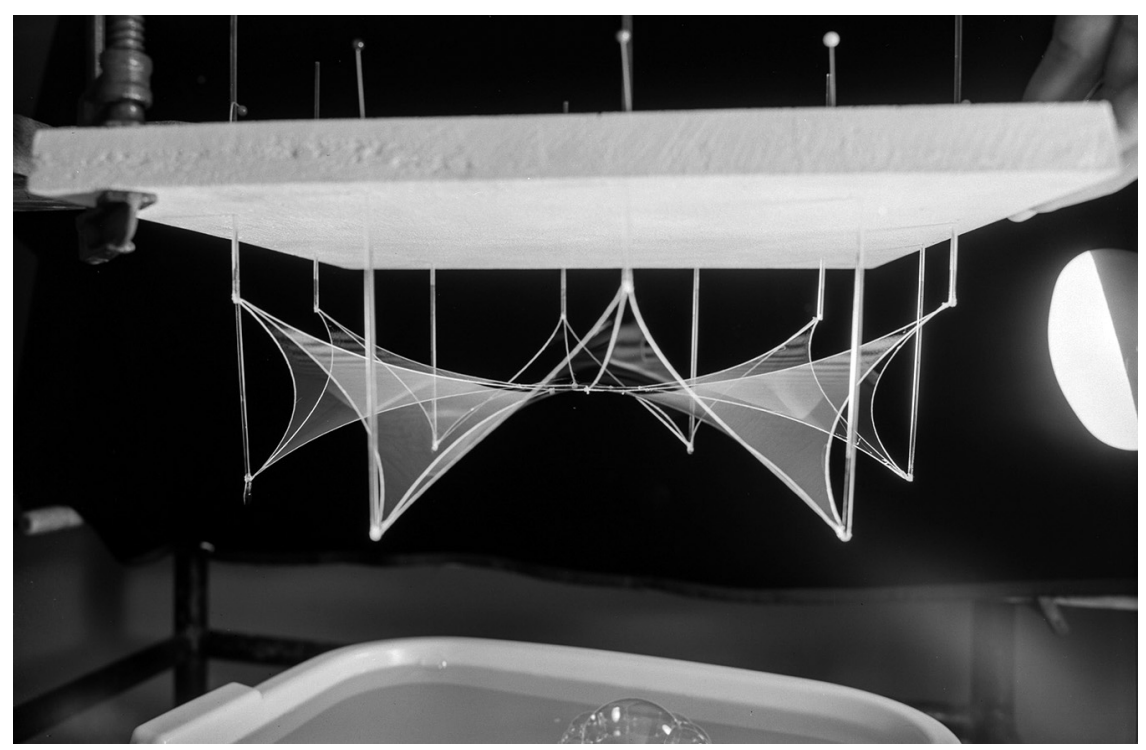

Ill. 8 : Maquette de bulles de savon du Tanzbrunnen (pavillon de danse), Entwicklungsstätte für den Leichtbau EL (Centre de développement pour la construction légère), Berlin 1957. (C) saai / Archive for Architecture and Civil Engineering, Karlsruhe Institute for Technology.

mesure, un écran de projection et une caméra. Il devient évident ici que pour Otto la " technologie ${ }^{16}$ " implique toujours une " technologie multimédia ». Il se soumet constamment aux lois physiques des surfaces membranaires, recherche systématiquement leurs propriétés géométriques et explore avec exactitude les possibilités des méthodes de construction respectives à l'aide de techniques médiatiques - dans le sens de Friedrich Dessauer, la sphère technique apparaît ici comme "étant réelle et devenant réelle par des idées ". La maquette s'inscrit dans un ensemble technique au sein duquel elle est mise en ouvre et sans lequel elle ne pourrait pas exister. En d'autres mots, la maquette et la machine deviennent acteurs du même système expérimental physique. La technologie de modélisation, de dessin et de prise de mesure, de même que la méthodologie d'évaluation constituent la base d'une culture expérimentale par la machine, qui implique un étalonnage constant de la coordination œil/main ; cette culture implique également l'observation scientifique, des compétences techniques, et un auto-ajustement manuel et intellectuel dans lequel la conception est à la fois une source de production individuelle de connaissances et un point de départ pour un discours collectif sur l'avenir de la discipline (ill. 8). Cela peut sembler paradoxal, mais une grande partie du potentiel épistémique de ces maquettes réside dans la temporalité et la fragilité du matériau luimême. Dans une certaine mesure, la productivité de ces montages expérimentaux repose sur le fait qu'elle n'exclut pas les dysfonctionnements, qui font plutôt partie 
intégrante de leur fonctionnement. En d'autres termes : Otto voit dans ses maquettes des indicateurs culturels qui vont au-delà de la sensation purement physique de l'objet individuel dans leur potentiel théorique et leur sens culturel et peuvent devenir le point d'origine symbolique d'une architecture libérée de ses contraintes.

\section{Esthétique opératoire}

Les maquettes architecturales d'Otto - si l'on peut encore parler ici de maquettes au sens classique - fonctionnent non seulement comme des objets statiques, mais aussi comme des objets dynamiques, c'est-à-dire comme des modèles de processus de l'environnement généré par la technologie des outils de médiatisation. Dans toute leur fragilité poétique, les maquettes de Frei Otto racontent l'histoire d'une esthétique opératoire qui oscille entre la précision des instruments scientifiques et l'imagination des œuvres d'art. Une fois de plus, la reformulation de l'histoire de la maquette architecturale fait débat. Si on se propose de raconter l'histoire architecturale, traditionnellement comprise comme l'histoire du bâtiment et du style, également comme une histoire culturelle des processus, des pratiques et des opérations de conception, et si on considère donc le changement d'optique de "produit du sens " vers la "production de sens " comme une lecture de l'architecture fondamentalement différente et cependant de signification équivalente, alors les maquettes expérimentales de Otto Frei offrent un point de départ fascinant.

Georg VRACHLIOTIS

Professeur de théorie architecturale et directeur des archives d'architecture du Karlsruhe Institute of Technology (KIT) 
